\title{
Synthesis, characterization, and antimicrobial activities of sulfonated chitosan
}

Zhimin Sun, Changgu Shi, Xiangyang Wang, Qiang Fang, Jianying Huang*

Key Laboratory of Fruits and Vegetables Postharvest and Processing Technology

Research of Zhejiang Province, College of Food Science and Biotechnology, Zhejiang

Gongshang University, Hangzhou 310018, P. R. China

Abstract: Sulfonated chitosan (SCS) was prepared via a facile chemical procedure by using 1,3-propane sultone attached to the backbone of chitosan. The resulting chitosan derivative was characterized by Fourier transform-infrared spectroscopy, nuclear magnetic resonance spectroscopy, gel permeation chromatography, elemental analysis, and thermo gravimetric analysis. The antibacterial experiments demonstrated that SCS exhibited higher antibacterial activities against Escherichia coli and Staphylococcus aureus with the minimum inhibitory concentration (MIC) of 0.13 $\mathrm{mg} / \mathrm{mL}$ and $2.00 \mathrm{mg} / \mathrm{mL}$ than those of water-soluble chitosan (WCS) with MIC of $0.50 \mathrm{mg} / \mathrm{mL}$ and $4.00 \mathrm{mg} / \mathrm{mL}$. However, different antifungal activities of SCS against Arthrinium sacchari (MIC, $64.00 \mathrm{mg} / \mathrm{mL}$ ) and Botrytis cinerea (MIC, $0.25 \mathrm{mg} / \mathrm{mL}$ ) were observed. Scanning electron microscopy revealed that WCS and SCS could damage and deform the structure of fungal hyphae with different action modes. It is worth mentioning that SCS exhibited selective antifungal activity, which was an important parameter to control its biological performance in many fields.

Keywords: Chitosan; Antimicrobial activity; Scanning electron microscopy; Arthrinium sacchari.

${ }^{*}$ Corresponding author: Tel: 86-571-2887-7777, E-mail:huangjy@zjgsu.edu.cn. 


\section{Introduction}

Chitosan, partially deacetylated derivative of chitin, is well known to be inexpensive, nontoxic, biocompatible and efficiently antimicrobial material (Pillai, Paul \& Sharma, 2009; Meng, Yang, Kennedy \& Tian, 2010). It has been widely utilized in biomedical, agricultural and food industrial. However, the remarkably poor solubility of chitosan due to the high crystallinity induced by hydrogen bonds and acetamido groups limits its application. Thus, it is necessary to modify the structure of chitosan to create derivatives with improved properties. Although chemical modification of chitosan to generate new bio-functional materials has achieved success (Zhang, Geng, Jiang, Li \& Huang, 2012; Geng, Yang, Huang, Zhang \& Wang, 2013; Jia, Duan, Fang, Wang \& Huang, 2016; Li, Duan, Huang \& Zheng, 2016), a strategy to reduce the positive charges of protonated amino groups presented on the polymeric surface, especially the introduction of sulfonate groups $\left(\mathrm{RSO}_{3}{ }^{-}\right)$on the chitosan chain, is still of great interest.

Anionic polysaccharides, in which their structures include sulfate, exhibit good biological activity and biocompatibility. For instance, heparin (HP), a linear biopolymer that contains sulfated $D$-glucosamine units, can be used to form polyelectrolyte complexes with cationic polymers at specific conditions of $\mathrm{pH}$ (Bueno et al., 2015; Martins, Pereira, Fajardo, Rubira \& Muniz, 2011; Fu, Ji, Yuan \& Shen, 2005) and act indirectly in the blood coagulation mechanism to stimulate cellular growth, as well as exhibit antibacterial effects (Bueno et al., 2015). Chondroitin sulfate $(\mathrm{ChS})$, another polysaccharide containing sulfonate groups, is an 
ingredient in health foods for the treatment of symptoms related to arthritis and cartilage repair because negatively charged $\mathrm{ChS}$ is capable of electrostatic interaction with positively charged growth factors to stabilize and prevent degradation of the growth factors in medium (Cai, Liu, Shu \& Prestwich, 2005; Lim et al., 2011).

A few useful matrixes containing chitosan and different anionic natural polyelectrolytes, such as heparin (Liu, Jiao, Liu \& Zhang, 2007) and chondroitin sulfate (Yeh, Cheng, Hu, Huang \& Young, 2011) have been developed for biocompatible applications and environmentally sensitive bio-materials (Tsai et al., 2015). However, ChS-CS nanoparticles are expected to be formed under acidic conditions ( $\mathrm{pH}$ below 6.5), limiting their applications in the delivery of acid-sensitive proteins and drugs. The presence of reactive hydroxyl and amino groups of chitosan enables physicochemical modifications to its structure thus obtaining versatile functional properties. Jayakumar et al. (Jayakumar, Nwe, Tokura \& Tamura, 2007) have well reviewed sulfated chitin and chitosan and their synthetic methods and potential applications. For example, the introduction of sulfonate groups into chitosan chain could improve its biological and chemical properties, such as blood anticoagulant activity (Suwan et al., 2009), antioxidant activity (Huang, Mendis \& Kim, 2005), hemagglutination inhibition, adsorption of metal ions (Ruiz, Sastre \& Guibal, 2003), antimicrobial activity (Zhong, Li, Xing \& Liu, 2009), as well as anti HIV-1 activity (Nishimura et al., 1998). Sulfonated chitosan (0.63\% sulfur content) exhibited antibacterial activity against Shigella dysenteriae, Aeromonas hydrophila, Salmonella typhimurium, and Bacillus cereus for the application in oyster 
preservation (Chen, Liau \& Tsai, 1998).

However, there still few information on the antimicrobial activities of SCS obtained by a facile chemical substitution with 1,3-propane sultone, including the antifungal activities against $A$. sacchari and $B$. cinerea. The objective of this study is to introduce sulfate group to the backbone of chitosan and to investigate the antimicrobial activity of the resulting anionic chitosan. Herein, sulfonic group was introduced into chitosan by nucleophilic substitution to afford $N$-(3-sulfonic) chitosan (sulfonated chitosan, SCS). The resulting anionic materials were characterized by Fourier transform-infrared spectroscopy (FT-IR), ${ }^{1} \mathrm{H}$ and ${ }^{13} \mathrm{C}$ nuclear magnetic resonance (NMR) spectroscopy, gel permeation chromatography (GPC), elemental analysis (EA), and thermo gravimetric analysis (TGA). Antimicrobial activities of sulfonated chitosan against E. coli, S. aureus, and A. sacchari, B. cinerea were further assessed and compared with that of water-soluble chitosan, followed the morphological hyphae changes investigation using scanning electron microscopy (SEM) techniques.

2. Materials and methods

\subsection{Materials}

The chitosan (CS) with the deacetylation of $91 \%$ and a centipoise viscosity of 60 $\mathrm{mPa} \cdot \mathrm{s}\left(1 \%, 20{ }^{\circ} \mathrm{C}\right)$ and water-soluble chitosan (WCS, water-soluble chitosan hydrochloride) with the deacetylation of $92 \%$ and a centipoise viscosity of $22 \mathrm{mPa} \cdot \mathrm{s}$

$\left(1 \%, 20{ }^{\circ} \mathrm{C}\right)$ used in the experiments were purchased from Qingdao Honghai Biotechnology Company (Shandong, China). Nutrient broth (NB), plate count agar 
(PCA), potato dextrose broth (PDB), and potato dextrose agar (PDA) were purchased from Hangzhou Microbial Reagent Co., Ltd. (Zhejiang, China). Other reagents and solvents were purchased from Aladin Reagent Co., Ltd. (Shanghai, China) and were used directly without further purification.

Microorganisms

Escherichia coli (E. coli, CICC 21524) and Staphylococcus aureus (S. aureus, CICC 10384) used in this study were purchased from China Center of Industrial Culture Collection (Beijing, China). Botryis Cinerea (B. cinerea, 3.611) was purchased from Beijing Yudingxinjie Technology Co., Ltd. (Beijing, China). Arthrinium sacchari (A. saccahri, KC354569) strain was isolated from deteriorated blueberries and identified by Sangon Biotech Co., Ltd. (Shanghai, China).

2.2 Synthesis of sulfonated chitosan (SCS)

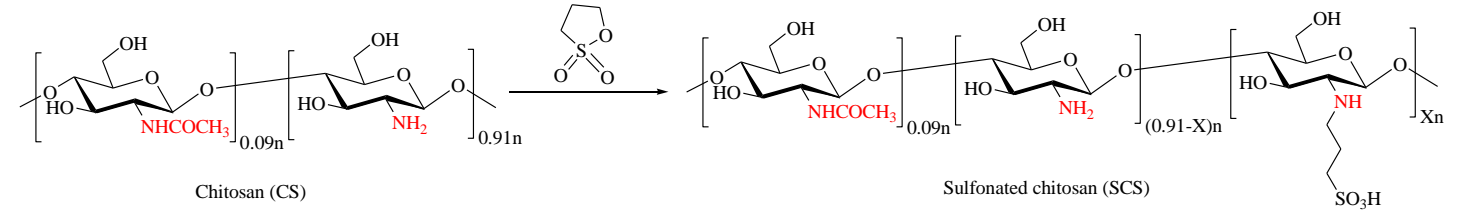

Scheme 1. Synthesis of sulfonated chitosan (SCS).

As shown in Scheme 1, SCS was prepared by modifying CS using 1,3-propane sultone according to the method described by Rwei and Lien (Rwei \& Lien, 2014) with minor modification. One gram of CS was completely dissolved in $80 \mathrm{~mL}$ of 2 wt $\%$ aqueous acetic acid solution. Afterwards, 2 grams of 1,3-propane sultone was added dropwise into CS solution under nitrogen atmosphere, and the result mixture was stirred at $60{ }^{\circ} \mathrm{C}$ for $6 \mathrm{~h}$. The solute was then poured into cold acetone with vigorous stirring to precipitate the white product. After completely washing with methanol to remove un-reacted 1,3-propane sultone, the solid was dried in an oven for 
$4 \mathrm{~h}$ to give SCS as a white powder.

\subsection{Characterization}

Fourier transform infrared spectroscopy (FT-IR) spectra were recorded on a Nicolet Nexus 870 spectrometer with $\mathrm{KBr}$ pellets. ${ }^{1} \mathrm{H}$ and ${ }^{13} \mathrm{C}$ NMR spectra were recorded at $343 \mathrm{~K}$ on a Bruker AVANCE DRX 500 (Bruker Bios pin, Rheinstetten, Germany). Molecular weight distributions were measured by a gel permeation chromatography (GPC) system equipped with a LC-20AD HPLC pump, a RID-20A RI detector, a Shodex SUGAR KS-804 linear chromatographic column in a CTO-20A thermostatic oven. GPC measurements were carried out at $60{ }^{\circ} \mathrm{C}$ using an ultrapure water solution as the eluent with a flow rate of $1.0 \mathrm{~mL} / \mathrm{min}$. The system was calibrated with Pullulan standards. Elemental analysis was conducted using an Element Vario MICRO cube elemental analyzer (Elementar, Germany), with a carrier gas (He, at a flow rate of 100 $\mathrm{mL} \cdot \mathrm{min}^{-1}$ ) at a combustion temperature of $1000{ }^{\circ} \mathrm{C}$ using the solid samples. The degree of sulfonation (DS) was determined by the N/S ratio based on the result of elemental analysis. DS was calculated according to Eq. (1) described by Rwei and Lien (Rwei \& Lien, 2014).

$\mathrm{N} / \mathrm{S}=14 \times(0.09 \times 1+(0.91-\mathrm{X}) \times 1+\mathrm{X} \times 1) / 32 \times \mathrm{X}, \mathrm{DS}(\%)=\mathrm{X} \times 100 \%$

Where $14 \mathrm{~g} / \mathrm{mol}$ is the molar mass of $\mathrm{N}$ and $32 \mathrm{~g} / \mathrm{mol}$ for $\mathrm{S}$, the deacetylation of chitosan is $91 \%$.

The thermo gravimetric analysis (TGA) was performed using a USA TA thermal analyzer Model Q50, at a heating rate of $10{ }^{\circ} \mathrm{C} \cdot \mathrm{min}^{-1}$, from room temperature up to $600{ }^{\circ} \mathrm{C}$ under an argon atmosphere.

2.4. Antibacterial activities of WCS and SCS against E. coli and S. aureus

2.4.1 Minimal inhibitory concentrations (MIC)

The MIC was determined according to a modified turbidimetric method in previous 
report (Geng, Yang, Huang, Zhang \& Wang, 2013). Polymers were dissolved in sterile nutrient broth, respectively, to prepare different concentration solutions. After autoclaving at $121{ }^{\circ} \mathrm{C}$ for $15 \mathrm{~min}$, samples were inoculated with $100 \mu \mathrm{L}$ of the freshly prepared bacteria suspension $\left(1 \times 10^{6} \mathrm{CFU} / \mathrm{mL}\right)$ in 96 well-microtitre plates and incubated at $37^{\circ} \mathrm{C}$ for $24 \mathrm{~h}$. The growth of bacteria was determined by optical density, as measured at $600 \mathrm{~nm}$ using a Microplate reader (M200, TECAN, Switzerland). The lowest concentration of samples inhibited the growth of bacteria was considered as MIC.

2.4.2 Minimal bactericidal concentration (MBC)

MBC was determined by subculturing the test dilution of minimum inhibitory concentration (MIC) value onto plate count agar (PCA) and incubating for 18-24 h according to the method described by Gonil et al. (Gonil et al., 2011). The highest dilution that yielded no single bacterial colony on a solid medium was taken as MBC. All experiments were performed in triplicate.

2.5 Antifungal activities of WCS and SCS against A. sacchari and B. cinerea

\subsubsection{Preparation of fungal spore suspension}

The fungi were cultured on potato dextrose agar (PDA) for seven days to achieve sporulation. Spores were then harvested by pouring sterile water into the slant and stirring with a vortex for 40 seconds. The suspensions of spores and mycelia were filtered through cotton. The spore concentrations were adjusted to $10^{5} \mathrm{CFU} / \mathrm{mL}$ using a hemocytometer under $400 \times$ maginification via inversed fluorescent microscopy (Nikon, Eclipse, TE 2000-S) (Jia, Duan, Fang, Wang \& Huang, 2016).

2.5.2 Minimal inhibitory concentration (MIC) and minimal fungicidal concentration 
(MFC)

The MIC values were determined using broth dilution assay. Different concentration stock solutions of WCS and SCS were prepared via dissolving in PDB solution at $\mathrm{pH}$ 6.3 by 2 -fold serial dilution. After autoclaving at $121{ }^{\circ} \mathrm{C}$ for $15 \mathrm{~min}$, the stock solutions were inoculated with $100 \mu \mathrm{L}$ fungal spore suspension (approximately $10^{5}$ $\mathrm{CFU} / \mathrm{mL}$ ). The cells were then incubated at $28{ }^{\circ} \mathrm{C}$ for 5 days. MIC was defined as the lowest concentration of the antifungal agent that prevented visible growth in comparison to the growth in control (PDB solution). Samples (100 $\mu \mathrm{L})$ were removed from each clear tube (from MIC determination) and spotted on petri dishes containing PDA. The plates were incubated for 5-7 days at $28{ }^{\circ} \mathrm{C}$. MFC was defined as the lowest concentration of antifungal agent which exhibited no visible growth after incubation (James Bound, Murthy \& Srinivas, 2016; Sajomsang, Gonil, Saesoo \& Ovatlarnporn, 2012).

\subsubsection{Mycelial growth inhibition}

The effect on mycelial growth of the fungi was assessed according to the method described in previous literature (Jia, Duan, Fang, Wang \& Huang, 2016). Mycelial stipitipellis (7 $\mathrm{mm}$ in diameter) from 1-week-old fungal cultures were placed in the center of $90 \mathrm{~mm}$ Petri dishes containing $20 \mathrm{~mL}$ PDA with various polymer concentrations, ranging from 0.13 to $16 \mathrm{mg} / \mathrm{mL}$, and were then incubated at $28{ }^{\circ} \mathrm{C}$ in the dark. Mycelial growth was determined by measuring the colony diameter 7 days after inoculation. Each treatment was replicated thrice, and the experiment was repeated twice. The inhibition percentage of mycelial growth was calculated using Eq (2) and was expressed as a percentage:

Antifungal index $(\%)=\left(1-D_{a} / D_{b}\right) \times 100 \%$

Where $D_{a}$ is the diameter of the growth zone in the test plates, and $D_{b}$ is the diameter 
of the growth zone in the control plate.

\subsubsection{The morphology of mycelial growth}

The effect of WCS and SCS on A. sacchari mycelial growth was investigated by photographs taken through scanning electron microscope (SEM, SU8010, Hitachi, Japan) after inoculation on PDA at $28{ }^{\circ} \mathrm{C}$ for 7 days. Scanning electron microscopy was carried out in accordance to the method described by Jia et al. (Jia, Duan, Fang, Wang \& Huang, 2016). The hyphae $\left(9 \mathrm{~mm}^{2}\right)$ of $A$. sacchari were separately obtained from the media with WCS and SCS $(8 \mathrm{mg} / \mathrm{mL})$ and the control group. The specimens were fixed with $2.5 \%$ glutaraldehyde in phosphate buffer $(0.1 \mathrm{~mol} / \mathrm{L}, \mathrm{pH} 7.0)$ for more than $4 \mathrm{~h}$, washed with phosphate buffer $(0.1 \mathrm{~mol} / \mathrm{L}, \mathrm{pH} 7.0)$, post-fixed with $1 \%$ osmium tetroxide in phosphate buffer $(0.1 \mathrm{~mol} / \mathrm{L}, \mathrm{pH} 7.0)$ for $2 \mathrm{~h}$ and washed again with phosphate buffer $(0.1 \mathrm{~mol} / \mathrm{L}, \mathrm{pH} 7.0)$. The specimens were then dehydrated in a graded ethanol series $(30,50,70,80,90,95$, and $100 \%$, v/v) with 15-min incubation per concentration. Subsequently, the specimens were transferred to a critical point dryer to complete the drying process with carbon dioxide as the transition fluid. The samples were then viewed and photographed with SEM.

2.6 Statistical Analysis.

Analysis of variance (ANOVA) $(\mathrm{P}<0.05)$ was performed using SPSS software (Version 18.0, SPSS, Inc., Chicago, IL). The mean differences were established via Duncan's multiple range tests.

3. Results and discussion

3.1 Synthesis and characterization 
In this study, chitosan with the deacetylation of $91 \%$, a centipoise viscosity of $60 \mathrm{mPa} \cdot \mathrm{s}\left(1 \%, 20{ }^{\circ} \mathrm{C}\right)$ was used as the starting material. After reaction with 1,3-propane sultone, a novel sulfonated chitosan (SCS) with 86\% degree of sulfonation (Scheme 1) was furnished as a white solid, which is water-soluble due to its negatively charged surface, similar to heparin and carrageenan. Notably, since the ingredient 1,3-propane sultone is known as a potent human carcinogen, the SCS, via complete reaction and purification, is quite safe and environment friendly. The purified SCS is potential to become a water-born antimicrobial additive or film for applications in various fields (Rwei \& Lien, 2014).

FT-IR spectroscopy. The FT-IR spectra of CS and SCS were presented in Fig. 1. The characteristic peaks of CS (a) located at $3419 \mathrm{~cm}^{-1}$ stand for the stretching vibration of the hydroxyl group and amide group, and the peaks at 1661 and $1587 \mathrm{~cm}^{-1}$ represent the acetylated amino group of chitosan (amide I) bands. As shown in Fig. 1(b), the FT-IR spectrum of SCS exhibits intense adsorption at 1034, and $1192 \mathrm{~cm}^{-1}$, corresponding to the $\mathrm{S}=\mathrm{O}$ stretching vibration from the sulfonic acid group. While the strong peaks at $1635 \mathrm{~cm}^{-1}$ and $1525 \mathrm{~cm}^{-1}$ were attributed to the $\mathrm{C}=\mathrm{O}$ stretching vibration of the amide group and the $\mathrm{C}-\mathrm{N}-\mathrm{C}$ bending vibration of the SCS branch. These results are in agreement with the reports by Rwei et al. and Jeon et al. (Jeon, Cheedarala, Kee \& Oh, 2013; Rwei \& Lien, 2014). A comparison of the FT-IR spectrum of CS with that of SCS reveals the differences between their respective absorbance bands, indicating that the sulfonation reaction occurred on the 2-amine groups. 


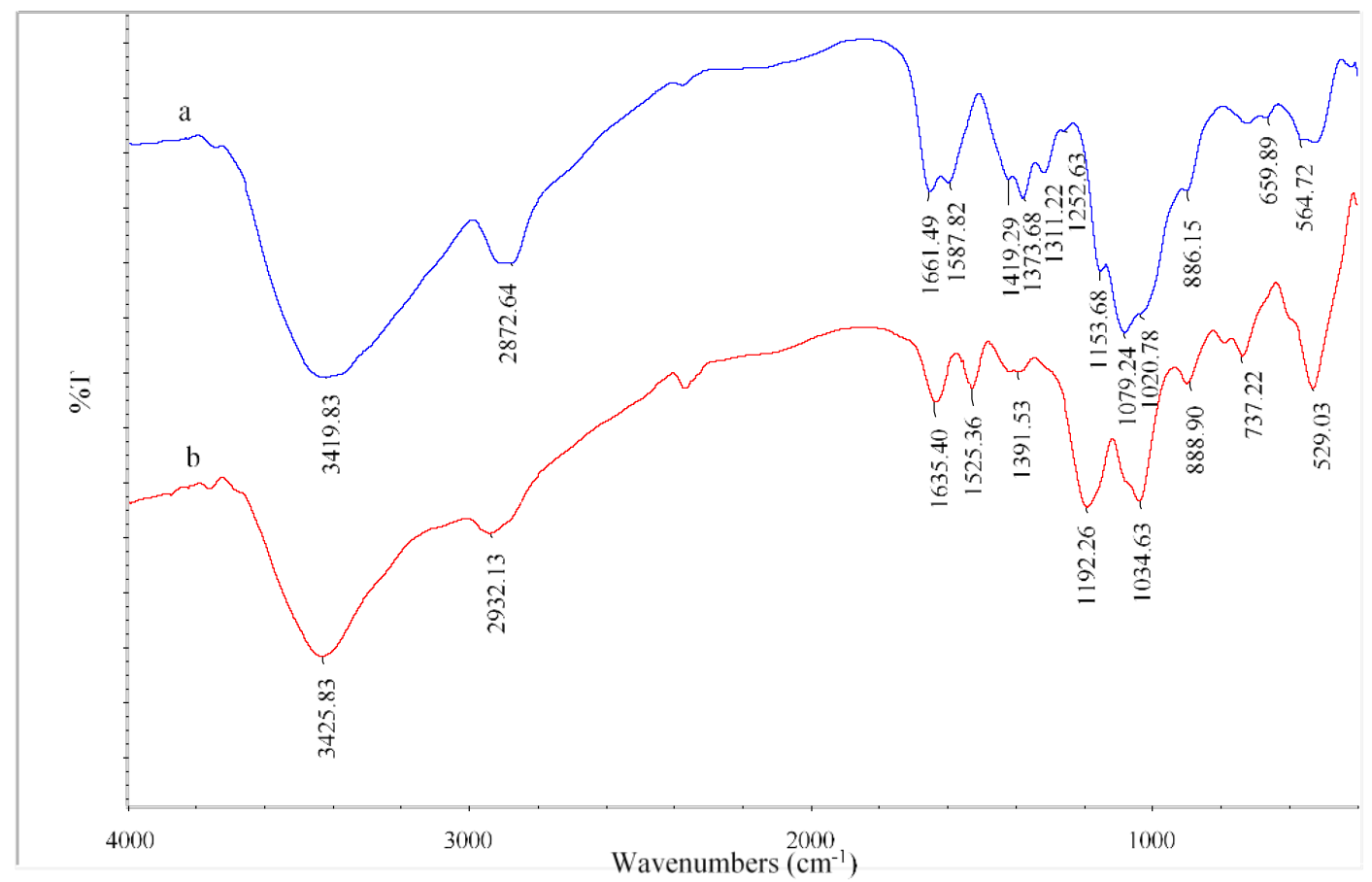

Fig. 1. FT-IR spectra of (a) CS and (b) SCS.

The ${ }^{1} \mathrm{H}-\mathrm{NMR}$ and ${ }^{13} \mathrm{C}-\mathrm{NMR}$ spectra of SCS in $\mathrm{D}_{2} \mathrm{O}$ solvent were presented in Fig. 2. The peaks at $1.92-1.98 \mathrm{ppm}$ were attributed to the presence of the acetamido methyl protons, while the singlet peaks at 2.82 and $3.03 \mathrm{ppm}$ was attributed to the $\mathrm{NH}_{2}$ of glucosamine and the $-\mathrm{CH}_{2}-$ in 1,3-propane sultone, respectively. The multiplets from 3.55 to $3.78 \mathrm{ppm}$ belong to the methenyl protons of glucosamine and $\mathrm{N}$-acetylated glucosamine. Accordingly, the signals at $\delta 22.2,22.4$, and $27.0 \mathrm{ppm}$ observed in the ${ }^{13} \mathrm{C}$-NMR spectrum were attributed to the carbons in 1,3-propane sultone and $N$-alkylated glucosamine residue, respectively, while the signal at $174.7 \mathrm{ppm}$ was assigned to the carbonyl carbon. Comparatively, the signals in ${ }^{13} \mathrm{C}-\mathrm{NMR}$ spectrum of $\mathrm{CS}$ in $\mathrm{D}_{2} \mathrm{O} / \mathrm{CF}_{3} \mathrm{COOD}$ were usually found at $\delta 55.6\left(\mathrm{C}_{2}\right), 60\left(\mathrm{C}_{6}\right), 70\left(\mathrm{C}_{3}\right), 75\left(\mathrm{C}_{5}\right)$, 76.5( $\left.\mathrm{C}_{4}\right)$ and 97.5( $\left.\mathrm{C}_{1}\right)$ ppm (Dung, Milas, Rinaudo \& Desbrières, 1994; Zhang, Ping, Zhang \& Shen, 2003). 


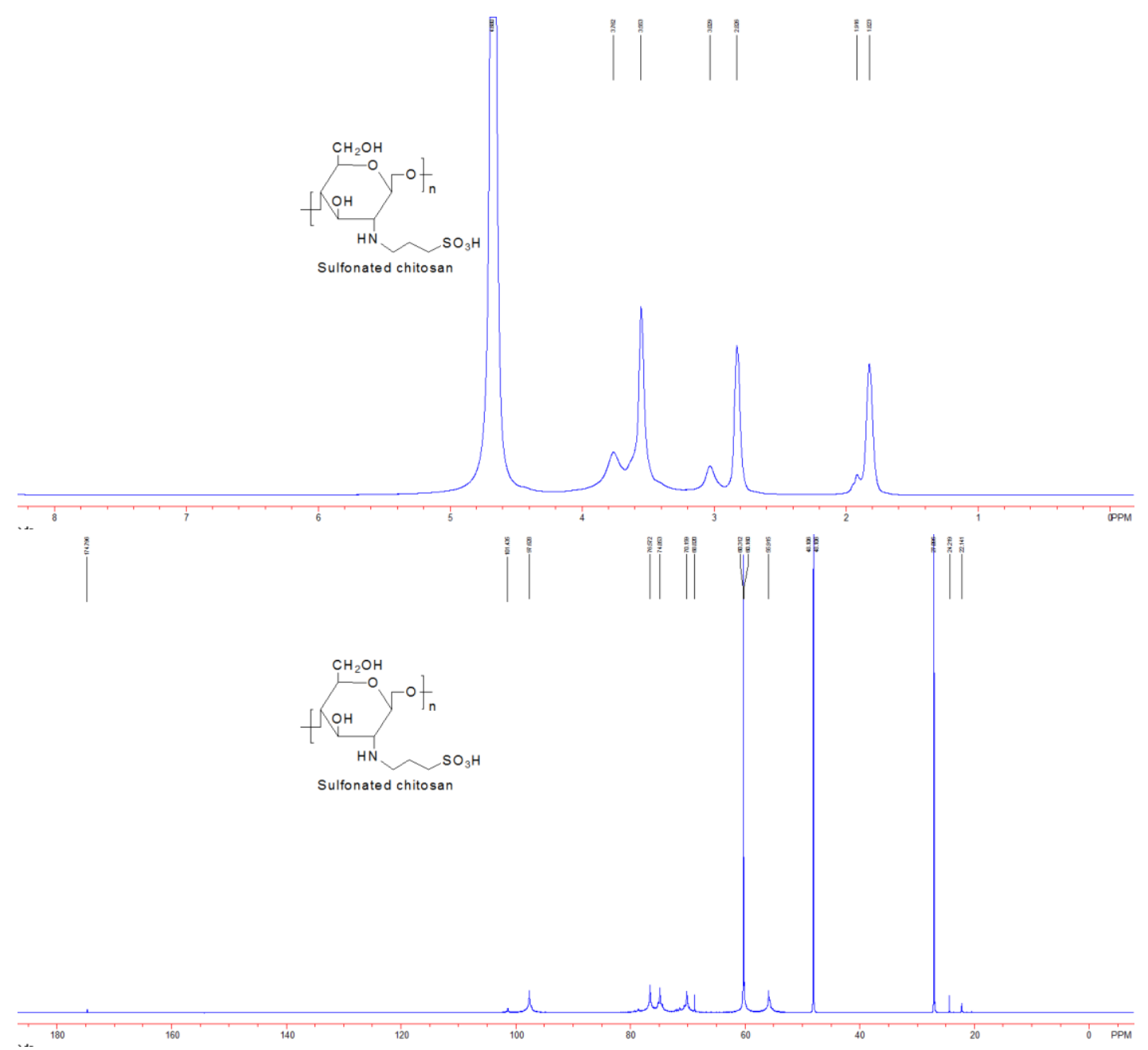

Fig. 2. ${ }^{1} \mathrm{H}-\mathrm{NMR}$ and ${ }^{13} \mathrm{C}-\mathrm{NMR}$ spectra of SCS in $\mathrm{D}_{2} \mathrm{O}$.

Gel permeation chromatography (GPC). Molecular weight of biopolymers is very important for industrial and scientific uses since the polymers with different molecular weight usually have different functions (Aranaz et al., 2009; Zhang, Geng, Jiang, Li \& Huang, 2012). Herein, the molecular weight distribution of each fraction, including its weight-average molecular weight value $(\mathrm{Mw})$ and number-average molecular weight value (Mn), was measured by GPC. As summarized in Table S1, the polydispersity (PD) was determined from the ratio of $\mathrm{Mw}$ to $\mathrm{Mn}$ to be 1.119 and 1.072 for WCS and SCS, respectively. The data in Table S1 also suggest that a mono-modal peak of SCS with a Mw is 31474, while that of WCS is 31061. 
Elemental analysis. After reaction, the nitrogen, carbon and hydrogen contents in SCS significantly decreased compared to the starting material CS (Table S2). The N/S ratio was $4.11 / 8.07$, so the degree of sulfonation of SCS was calculated to be $86 \%$, accordingly. The DS of SCS prepared herein is slightly increased, by comparison with the sulfonated chitosan in Rwei \& Lien's work (Rwei \& Lien, 2014), most probably due to the higher reaction temperature and prolonged reaction time.

Thermo gravimetric analysis. TG (a) and DTG (b) curves of CS, WCS and SCS were shown in Fig. S1. The TGA results of CS and WCS were similar to each other and both degraded in two stages. The first degradation stage of CS was started at $50{ }^{\circ} \mathrm{C}$ till $280{ }^{\circ} \mathrm{C}$, with weight loss about $4 \%$ which was due to the loss of solvent (water), while that of WCS was from $40{ }^{\circ} \mathrm{C}$ to $200{ }^{\circ} \mathrm{C}$, with weight loss of $6 \%$. From $280{ }^{\circ} \mathrm{C}$ to $600{ }^{\circ} \mathrm{C}$, the second stage of CS was explained as the degradation of CS skeleton, involved a weight loss around 57\%. In the case of WCS, the second degradation stage started at $200{ }^{\circ} \mathrm{C}$ till $550{ }^{\circ} \mathrm{C}$ with weight loss of $60 \%$. It was consistent with the results reported by Xiao et al. (Qin, Du \& Xiao, 2002). As compared to CS and WCS, SCS underwent three-stage decomposition: one water loss and two decomposition. The first stage ranged from $40{ }^{\circ} \mathrm{C}$ to $190{ }^{\circ} \mathrm{C}$, with a $4 \%$ loss of water molecules. The second stage occurred from $190{ }^{\circ} \mathrm{C}$ to $235{ }^{\circ} \mathrm{C}$, and the temperature of maximum decomposition rate $\left(\mathrm{T}_{\max }\right)$ was $212{ }^{\circ} \mathrm{C} . \mathrm{T}_{\max }$ of SCS is equal to that of $\mathrm{WCS}$, but lower than that of $\mathrm{CS}\left(\mathrm{T}_{\max }, 299^{\circ} \mathrm{C}\right)$. It followed with a further $25 \%$ weight loss which is attributed to the loss of sulfonic acid groups on the chitosan polymer. The third stage of deposition ended at $510{ }^{\circ} \mathrm{C}$ with weight loss of $37 \%$. The results is similar to those reported by Lien et al. (Tsai, Wang, Lin \& Lien, 2010) and Oh et al. (Jeon, Cheedarala, Kee \& Oh, 2013).

3.2. Antibacterial activity of WCS and SCS against E. coli and S. aureus 
The antibacterial activities of SCS against E. coli and S. aureus were assessed by turbidity method. Simultaneously, the same method was performed on WCS for comparison. The results were presented in Table 1. The MIC values of WCS and SCS were $0.50,0.13 \mathrm{mg} / \mathrm{mL}$ for $E$. coli, and $4.00,2.00 \mathrm{mg} / \mathrm{mL}$ for $S$. aureus, respectively (Fig. 3), while the MBC values of WCS and SCS were $2.00,1.00 \mathrm{mg} / \mathrm{mL}$ for E. coli, and $32.00,16.00 \mathrm{mg} / \mathrm{mL}$ for $S$. aureus, respectively (Fig. S2). The antibacterial activities of SCS against E. coli and $S$. aureus are significantly higher than that of WCS. Tan et al. reported that 3,6-O-sulfated chitosan-modified surface loaded with lysozyme was capable of killing almost all of the $E$. coli cells attached to the material with very high hydrolysis activity for bacterial cell wall components (Tan, Wang, Wang, Chen, Yuan \& Chen, 2014; Zhong, Li, Xing \& Liu, 2009). The results indicated that the introduction of alkyl sulfonic groups into chitosan backbone could improve its antibacterial activities. Moreover, the antibacterial activity of SCS was more effective against $E$. coli than $S$. aureus, suggesting that this chitosan derivative showed stronger antibacterial activity against Gram-negative than Gram-positive bacteria, which was accordance with the study of Zhong et al. (Zhong, Li, Xing \& Liu, 2009). 

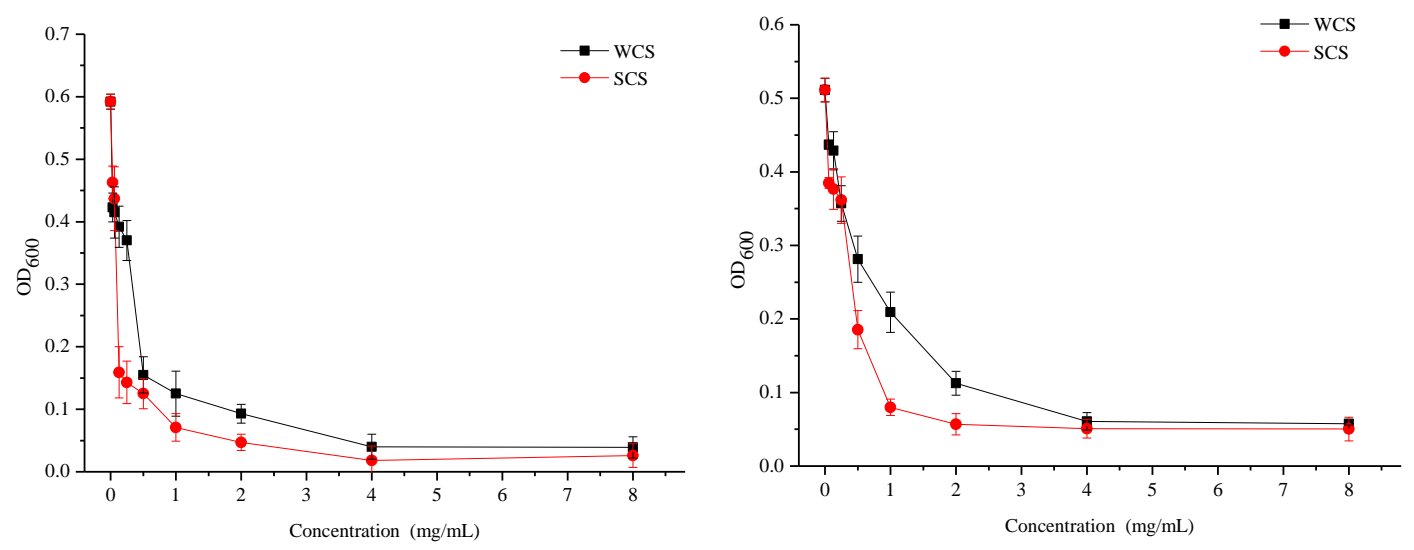

Fig. 3. Antibacterial effect of WCS and SCS on the growth of E. coli (left) and $S$. aureus (right) by turbidity.

Table 1. The MIC and MBC of WCS, SCS against E. coli and S. aureus

\begin{tabular}{llllll}
\hline \multirow{2}{*}{ Microorganisms } & \multicolumn{2}{l}{ WCS $(\mathrm{mg} / \mathrm{mL})$} & & \multicolumn{2}{l}{ SCS $(\mathrm{mg} / \mathrm{mL})$} \\
\cline { 2 - 3 } \cline { 6 - 7 } \cline { 6 - 7 } & MIC & MBC & & MIC & MBC \\
S. aureus & 0.50 & 2.00 & & 0.13 & 1.00 \\
\hline
\end{tabular}

3.3. Antifungal activity of WCS and SCS against $A$. sacchari and B. cinerea

A. sacchari easily infects durum wheat leading damping-off with the characteristic dark brown or violet lesions in infected tissues (Mavragani, Abdellatif, Mcconkey, Hamel \& Vujanovic, 2007). Herein, the effect of WCS and SCS against A. sacchari and $B$. cinerea were assessed. The results of MIC and MFC values were shown in Table 2. It was clearly observed that $B$. cinerea is highly susceptible to both WCS and SCS (MIC $0.13 \mathrm{mg} / \mathrm{mL}$ and $0.25 \mathrm{mg} / \mathrm{mL}$, MFC 0.13 and $0.50 \mathrm{mg} / \mathrm{mL}$, respectively) (Table 2 and Fig. S4, S5). However, the MIC of SCS against A. sacchari was 64 $\mathrm{mg} / \mathrm{mL}$, while the MFC of SCS reached up to $128 \mathrm{mg} / \mathrm{mL}$ (Table 2 and Fig. S3, S5).

Table 2. The MIC and MFC of WCS, SCS against A. sacchari and B. cinerea

\begin{tabular}{|c|c|c|c|c|}
\hline \multirow{2}{*}{ Microorganisms } & \multicolumn{2}{|c|}{ WCS $(\mathrm{mg} / \mathrm{mL})$} & \multicolumn{2}{|c|}{$\mathrm{SCS}(\mathrm{mg} / \mathrm{mL})$} \\
\hline & MIC & MFC & MIC & MFC \\
\hline A. sacchari & 16.00 & 16.00 & 64.00 & 128.00 \\
\hline B. cinerea & 0.13 & 0.13 & 0.25 & 0.50 \\
\hline
\end{tabular}

The mycelial growth inhibition of WCS and SCS against $A$. sacchari and B. cinerea were further investigated. As shown in Fig. 4, Fig. S6, and Fig. S7, the inhibitory 
effects of WCS and SCS are dose-dependent. A marked fungus radial growth inhibition of both WCS and SCS against $B$. cinerea was observed at a concentration of $4 \mathrm{mg} / \mathrm{mL}$ with 100\% inhibition (Fig. 4 and S7), indicating that both WCS and SCS exhibited high antifungal activity against $B$. cinerea, while the antifungal indexes of WCS and SCS against A. sacchari were $74.5 \%$ and $81.8 \%$, even at a concentration of $16 \mathrm{mg} / \mathrm{mL}$ (Fig. 4 and S6). These results of mycelial growth inhibition are correlated with the above-mentioned MIC and MFC determination, further illustrating that the effect of the antifungal activity of chitosan depends on the type of fungus (Gabriel, Tiera \& Tiera, 2015).
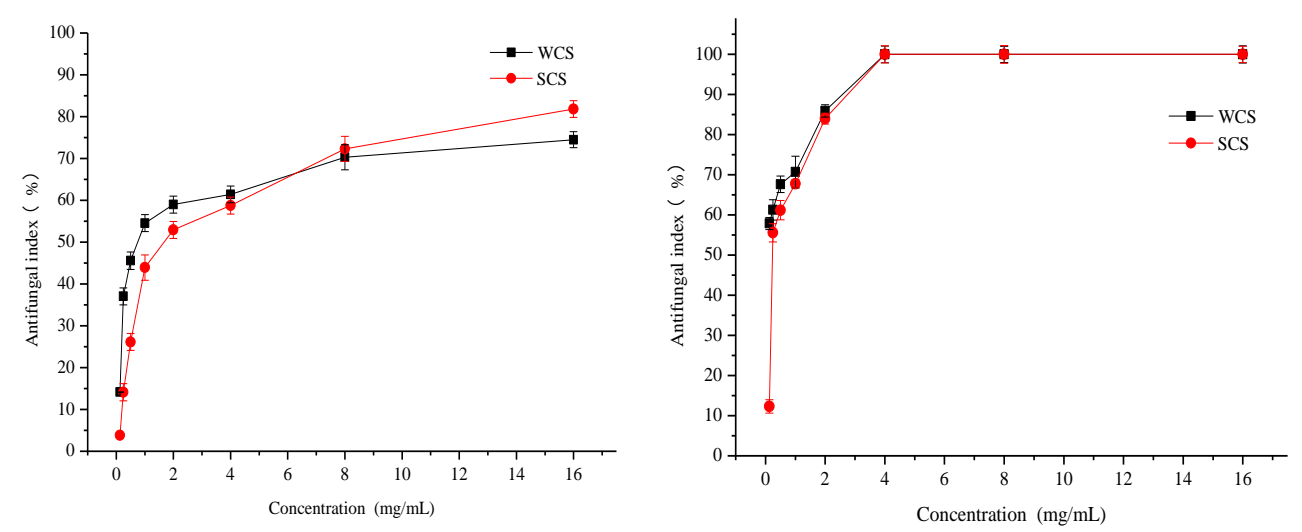

Fig. 4. Effect of WCS and SCS on mycelial growth of $A$. sacchari (left) and $B$. cinerea (right).

The significantly different effect of SCS against $A$. sacchari and B. cinerea (Table 2, Fig. 4, S6, and S7) is potential to the applications in many fields. For example, A. sacchari could help Termitomyces decompose lignocellulosic material, thus stimulating its growth by 58.6\% (Sawhasan, Worapong, Flegel \& Vinijsanun, 2012). Tsukada et al. reported that $A$. sacchari isolated from sponges and algae of marine possesses antiproliferation effects on human umbilical vein endothelial and human umbilical artery endothelial cells, which is a potential fungi for anticancer therapy 
(Tsukada et al., 2011). Therefore, the certain concentration of SCS that exhibited selective bactericidal activity could be used in specific medical and agricultural industries. Miao et al. reported that Arthrinium. c.f. saccharicola isolated from seawater might produce more defensive compounds (such as antibacterial or other bioactive compounds) under stress condition. The existence of WCS and SCS in PDA media may cause a stress condition for the growth of $A$. sacchari, this finding can well explain the reason of the poor antifungal effect of WCS and SCS against $A$. sacchari.

Morphological Images. Although different mechanisms of the antimicrobial activity of chitosan have been proposed, the exact mechanism remains controversial. Severe morphological changes, including abnormal shapes and swelling mycelia (Oliveira Junior, Melo \& Franco, 2012) and large vesicles or empty cells devoid of cytoplasm in the mycelium (Barka, Eullaffroy, Clément \& Vernet, 2004) have been explored, while no morphological changes in chitosan-treated B. cinerea were also reported by Ghaouth et al. and Mohamed et al. (Ghaouth, Arul, Asselin \& Benhamou, 1992; Mohamed, Sabaa, El-Ghandour, Abdel-Aziz \& Abdel-Gawad, 2013). In view of above-mentioned diverging results reported by other researchers and the significant different inhibition effect of WCS and SCS against A. sacchari obtained herein, scanning electron microscopy was used to investigate the antifungal mode of WCS and SCS. As shown in Fig. $5 \mathrm{~A}_{1}-\mathrm{A}_{2}$ and S8A, a dense and smooth network of hyphae surrounded by an exopolymeric matrix and elongated hyphae was observed in the edge of untreated A. sacchari culture. Conversely, abnormal morphological hyphae, 
such as aggregation, excessive mycelial branching and unshaped hyphae, were observed in WCS- and SCS-treated A. sacchari (Fig. 5B $\mathrm{B}_{1}-\mathrm{B}_{2}$, S8B and Fig. $5 \mathrm{C}_{1}-\mathrm{C}_{2}$, S8C). In the case of WCS-treated A. sacchari, a large irregularly spherical and/or club-shaped cluster was obviously observed on the end of the deformed and twisted hyphae (Fig. $5 \mathrm{~B}_{1}-\mathrm{B}_{2}$ and $\mathrm{S} 8 \mathrm{~B}$ ), which is similar with pyridine chitosan-treated $B$. cinerea (Jia, Duan, Fang, Wang \& Huang, 2016). However, the different hyphae morphological micrograph was found in SCS-treated A. sacchari (Fig. $5 \mathrm{C}_{1}-\mathrm{C}_{2}, \mathrm{~S} 8 \mathrm{C}$ ), hyphae were obscure and wizzled, and looser than the control and WCS-treated $A$. sacchari, and showed less obvious club-shaped cluster in comparison with WCS-treated A. sacchari, inferring different antifungal action of WCS and SCS against A. sacchari. Abnormal shapes and swelling mycelia of pyridine chitosan-treated B. cinerea observed from SEM (Jia, Duan, Fang, Wang \& Huang, 2016) were similar with the WCS-treated A. sacchari, but significantly different from the SCS-treated A. sacchari. 


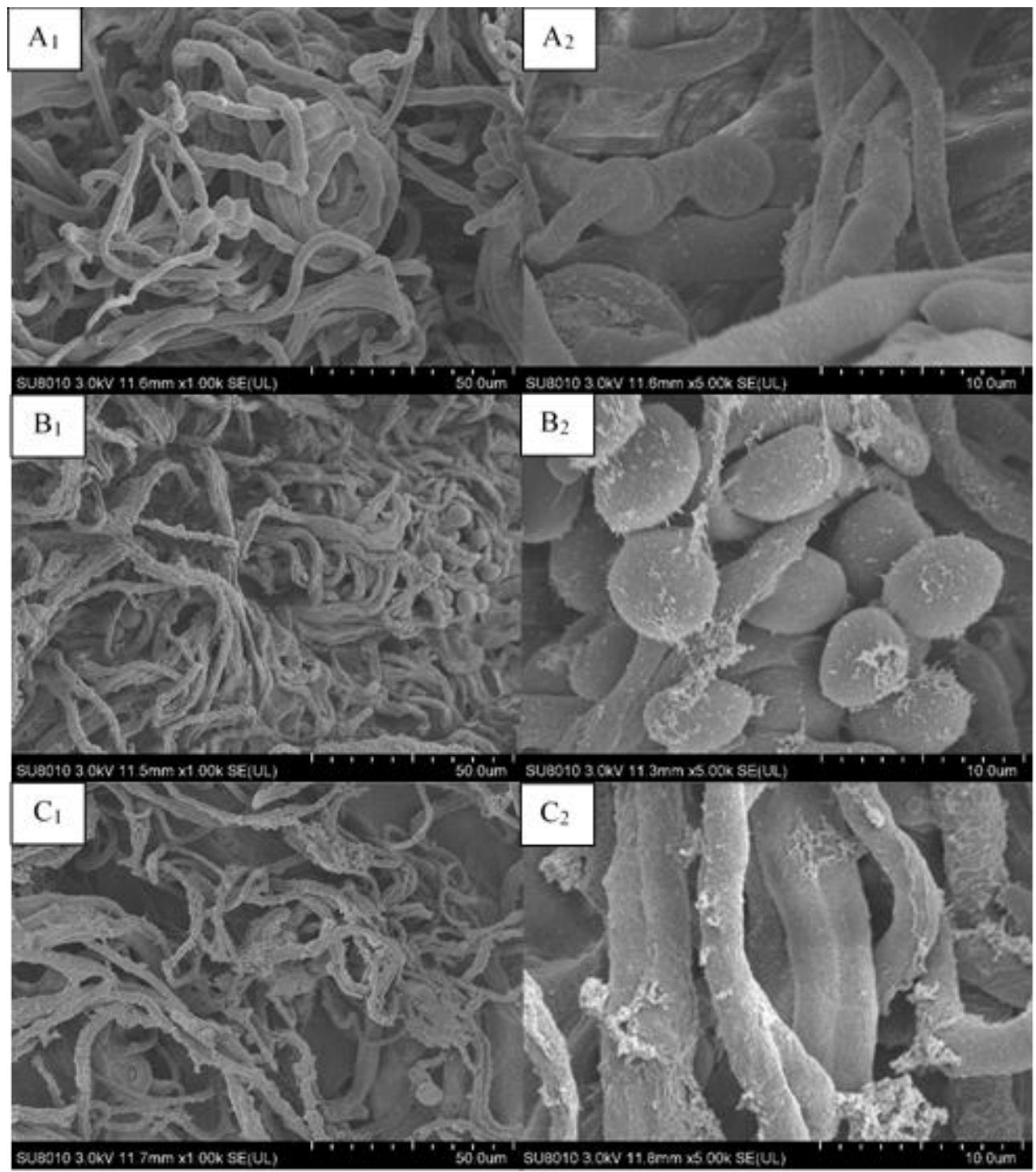

Fig. 5. Morphological micrographs of untreated A. sacchari $\left(\mathrm{A}_{1}-\mathrm{A}_{2}\right)$, WCS-treated $A$. sacchari $\left(\mathrm{B}_{1}-\mathrm{B}_{2}\right)$ and SCS-treated $A$. sacchari $\left(\mathrm{C}_{1}-\mathrm{C}_{2}\right)$ removed from 7-day-old PDA cultures as analyzed by SEM.

It was supposed that polycationic structure of chitosan in acidic media could interact with anionic components (i.e., proteins, phospholipids) of cell surface, thereby interfering their normal growth and metabolism (Fang, Li \& Dyc, 1994; Shahidi, Arachchi \& Jeon, 1999; Roller \& Covill, 1999; Bautista-Baños et al, 2006). Another possible mechanism is that chitosan chains may cross the cell membrane, interact with DNA, alter its conformation, leading to inhibition of the synthesis of mRNA and proteins (Sajomsang, Gonil, Saesoo \& Ovatlarnporn, 2012; Tan, Rui, Lin, Liu \& Tang, 2013).It is possible that the water-soluble SCS is able to penetrate cell walls and 
membranes interfering directly with the activity of enzymes responsible for growth of the fungi.

Abnormal shapes and swelling mycelia of pyridine chitosan-treated B. cinerea observed from SEM (Jia, Duan, Fang, Wang \& Huang, 2016) were similar with the WCS-treated A. sacchari, but significantly different from the SCS-treated A. sacchari.

\section{Conclusion}

Chemical bactericides and fungicides residues have caused much attention due to their non-biodegradability, toxicity, as well as serious public and environmental problems. In this study, a novel water-soluble sulfonated chitosan, as a kind of linear sulfated polysaccharide like carrageenans/furcelleran, was successfully synthesized by introducing 1,3-propane sultone to the amino group of chitosan under mild acidic conditions. The results demonstrated that the introduction of hydrophilic alkyl sulfonic acid group onto chitosan enhanced water solubility with decreased thermal stability. Different hyphae morphological changes and antifungal activities between WCS- and SCS-treated A. sacchari were found, suggesting that the fungal inhibition was chitsoan type- and fungi-dependent. The selective antimicrobial activity of SCS plays an important role in controlling its biological performance, thus exhibiting great potential applications in many fields.

\section{Acknowledgments}

This study was supported by the Natural Science Foundation of Zhejiang Province of China (Y3110204), the Technology Planning Project of Zhejiang Province of China (2011C12031), the National Science Foundation of China (21102129). We are grateful to Sangon Biotech (Shanghai) Co., Ltd. for DNA sequencing. 


\section{References:}

Aranaz, I., Mengibar, M., Harris, R., Panos, I., Miralles, B., Acosta, N., Galed, G., \& Heras, A. (2009).

Functional characterization of chitin and chitosan. Current Chemical Biology, 3(2), 203-230.

Barka, E. A., Eullaffroy, P., Clément, C., \& Vernet, G. (2004). Chitosan improves development, and protects Vitis vinifera L. against Botrytis cinerea. Plant Cell Reports, 22(8), 608-614.

Bautista-Baños, S., Hernández-Lauzardo, A. N., Valle, M. G. V., Hernández-López, M., Barka, E. A., Bosquez-Molina, E., \& Wilson, C. L. (2006). Chitosan as a potential natural compound to control pre and postharvest diseases of horticultural commodities. Crop Protection, 25(2), 108-118.

Bueno, P. V. A., Souza, P. R., Follmann, H. D. M., Pereira, A. G. B., Martins, A. F., Rubira, A. F., \& Muniz, E. C. (2015). N, N-Dimethyl chitosan/heparin polyelectrolyte complex vehicle for efficient heparin delivery. International Journal of Biological Macromolecules, 75, 186-191.

Cai, S., Liu, Y., Shu, X. Z., \& Prestwich, G. D. (2005). Injectable glycosaminoglycan hydrogels for controlled release of human basic fibroblast growth factor. Biomaterials, 26(30), 6054-6067.

Chen, C. S., Liau, W. Y., \& Tsai, G. J. (1998). Antibacterial effects of $N$-sulfonated and $N$-sulfobenzoyl chitosan and application to oyster preservation. Journal of Food Protection, 61(9), 1124-1128.

Dung, P. L., Milas, M., Rinaudo, M., \& Desbrières, J. (1994). Water soluble derivatives obtained by controlled chemical modifications of chitosan. Carbohydrate Polymers, 24(3), 209-214.

Fang, S. W., Li, C. F., \& Dyc, S. (1994). Antifungal activity of chitosan and its preservative effect on low-sugar candied kumquat. Journal of Food Protection, 57(2), 136-140.

Fu, J., Ji, J., Yuan, W., \& Shen, J. (2005). Construction of anti-adhesive and antibacterial multilayer films via layer-by-layer assembly of heparin and chitosan. Biomaterials, 26(33), 6684-6692.

Gabriel, J. S., Tiera, M. J., \& Tiera, V. A. (2015). Synthesis, characterization, and antifungal activities 
of amphiphilic derivatives of diethylaminoethyl chitosan against Aspergillus flavus. Journal of Agricultural and Food Chemistry, 63(24), 5725-5731.

Geng, X., Yang, R., Huang, J., Zhang, X., \& Wang, X. (2013). Evaluation antibacterial activity of quaternary-based chitin/chitosan derivatives in vitro. Journal of Food Science, 78(1), M90-M97.

Ghaouth, A. E., Arul, J., Asselin, A., \& Benhamou, N. (1992). Antifungal activity of chitosan on post-harvest pathogens: Induction of morphological and cytological alterations in Rhizopus. stolonifer. Mycological Research, 96(9), 769-779.

Gonil, P., Sajomsang, W., Ruktanonchai, U. R., Pimpha, N., Sramala, I., Nuchuchua, O., Saesoo, S., Chaleawlert-umpon, S., \& Puttipipatkhachorn, S. (2011). Novel quaternized chitosan containing $\beta$-cyclodextrin moiety:Synthesis, characterization and antimicrobial activity. Carbohydrate Polymers, 83(2), 905-913.

Huang, R., Mendis, E., \& Kim, S. K. (2005). Factors affecting the free radical scavenging behavior of chitosan sulfate. International Journal of Biological Macromolecules, 36(1-2), 120-127.

James Bound, D., Murthy, P. S., \& Srinivas, P. (2016). 2, 3-Dideoxyglucosides of selected terpene phenols and alcohols as potent antifungal compounds. Food Chemistry, 210, 371-380.

Jayakumar, R., Nwe, N., Tokura, S., \& Tamura, H. (2007). Sulfated chitin and chitosan as novel biomaterials. International Journal of Biological Macromolecules, 40(3), 175-181.

Jeon, J., Cheedarala, R. K., Kee, C., \& Oh, I. (2013). Dry-type artificial muscles based on pendent sulfonated chitosan and functionalized graphene oxide for greatly enhanced ionic interactions and mechanical stiffness. Advanced Functional Materials, 23(48), 6007-6018.

Jia, R., Duan, Y., Fang, Q., Wang, X., \& Huang, J. (2016). Pyridine-grafted chitosan derivative as an antifungal agent. Food Chemistry, 196, 381-387. 
Li, W., Duan, Y., Huang, J., \& Zheng, Q. (2016). Synthesis, antioxidant and cathepsin D inhibition activity of quaternary ammonium chitosan derivatives. Carbohydrate Polymers, 136, 884-891.

Lim, J. J., Hammoudi, T. M., Bratt-Leal, A. M., Hamilton, S. K., Kepple, K. L., Bloodworth, N. C., Mcdevitt, T. C., \& Temenoff, J. S. (2011). Development of nano- and microscale chondroitin sulfate particles for controlled growth factor delivery. Acta Biomaterialia, 7(3), 986-995.

Liu, Z., Jiao, Y., Liu, F., \& Zhang, Z. (2007). Heparin/chitosan nanoparticle carriers prepared by polyelectrolyte complexation. Journal of Biomedical Materials Research Part A, 83 (3), 806-812.

Martins, A. F., Pereira, A. G. B., Fajardo, A. R., Rubira, A. F., \& Muniz, E. C. (2011). Characterization of polyelectrolytes complexes based on $N, N, N$-trimethyl chitosan/heparin prepared at different $\mathrm{pH}$ conditions. Carbohydrate Polymers, 86(3), 1266-1272.

Mavragani, D. C., Abdellatif, L., Mcconkey, B., Hamel, C., \& Vujanovic, V. (2007). First Report of Damping-Off of Durum Wheat Caused by Arthrinium sacchari in the Semi-Arid Saskatchewan Fields. Plant Disease, 91(4), 469.

Meng, X., Yang, L., Kennedy, J. F., \& Tian, S. (2010). Effects of chitosan and oligochitosan on growth of two fungal pathogens and physiological properties in pear fruit. Carbohydrate Polymers, 81(1), 70-75.

Miao, L., Kwong, T. F. N., \& Qian, P. (2006). Effect of culture conditions on mycelial growth, antibacterial activity, and metabolite profiles of the marine-derived fungus Arthrinium c.f. saccharicola. Applied Microbiology and Biotechnology, 72(5), 1063-1073.

Mohamed, N. A., Sabaa, M. W., El-Ghandour, A. H., Abdel-Aziz, M. M., \& Abdel-Gawad, O. F. (2013). Quaternized $N$-substituted carboxymethyl chitosan derivatives as antimicrobial agents. International Journal of Biological Macromolecules, 60(9), 156-164. 
Nishimura, S., Kai, H., Shinada, K., Yoshida, T., Tokura, S., Kurita, K., Nakashima, H., Yamamoto, N., \& Uryu, T. (1998). Regioselective syntheses of sulfated polysaccharides: specific anti-HIV-1 activity of novel chitin sulfates. Carbohydrate Research, 306(3), 427-433.

Oliveira Junior, E. N. D., Melo, I. S. D., \& Franco, T. T. (2012). Changes in hyphal morphology due to chitosan treatment in some fungal species. Brazilian Archives of Biology \& Technology, 55(5), 637-646

Pillai, C. K. S., Paul, W., \& Sharma, C. P. (2009). Chitin and chitosan polymers: chemistry, solubility and fiber formation. Progress in Polymer Science, 34(7), 641-678.

Qin, C. Q., Du, Y. M., \& Xiao, L. (2002). Effect of hydrogen peroxide treatment on the molecular weight and structure of chitosan. Polymer Degradation and Stability, 76(2), 211-218.

Roller, S., \& Covill, N. (1999). The antifungal properties of chitosan in laboratory media and apple juice. International Journal of Food Microbiology, 47, 67-77.

Ruiz, M., Sastre, A., \& Guibal, E. (2003). Osmium and iridium sorption on chitosan derivatives. Solvent Extraction \& Ion Exchange, 21(2), 307-329.

Rwei, S., \& Lien, C. (2014). Synthesis and viscoelastic characterization of sulfonated chitosan solutions. Colloid and Polymer Science, 292(4), 785-795.

Sajomsang, W., Gonil, P., Saesoo, S., \& Ovatlarnporn, C. (2012). Antifungal property of quaternized chitosan and its derivatives. International Journal of Biological Macromolecules, 50(1), 263-269.

Sawhasan, P., Worapong, J., Flegel, T. W., \& Vinijsanun, T. (2012). Fungal partnerships stimulate growth of Termitomyces clypeatus stalk mycelium in vitro. World Journal of Microbiology and Biotechnology, 28(6), 2311-2318.

Shahidi, F., Arachchi, J. K. V., \& Jeon, Y. J. (1999). Food applications of chitin and chitosans. Trends in 
Food Science and Technology, 2, 37-51.

Suwan, J., Zhang, Z., Li, B., Vongchan, P., Meepowpan, P., Zhang, F., Mousa, S. A., Mousa, S., Premanode, B., Kongtawelert, P., \& Linhardt, R. J. (2009). Sulfonation of papain-treated chitosan and its mechanism for anticoagulant activity. Carbohydrate Research, 344(10), 1190-1196.

Tan, H., Rui, M., Lin, C., Liu, Z., \& Tang, T. (2013). Quaternized chitosan as an antimicrobial agent: antimicrobial activity, mechanism of action and biomedical applications in orthopedics. International Journal of Molecular Sciences, 14(1), 1854-1869.

Tan, M., Wang, H., Wang, Y., Chen, G., Yuan, L., \& Chen, H. (2014). Recyclable antibacterial material: silicon grafted with 3,6-O-sulfated chitosan and specifically bound by lysozyme. Journal of Materials Chemistry B, 2(5), 569-576.

Tsai, H. S., Wang, Y. Z., Lin, J. J., \& Lien, W. F. (2010). Preparation and properties of sulfopropyl chitosan derivatives with various sulfonation degree. Journal of Applied Polymer Science, 116(3), 1686-1693.

Tsai, T., Yen, H., Chen, C., Chen, Y., Young, Y., Cheng, K., Young, J., \& Hong, P. (2015). Novel protein-loaded chondroitin sulfate- $N$-[(2-hydroxy-3-trimethylammonium) propyl]chitosan nanoparticles with reverse zeta potential: preparation, characterization, and ex vivo assessment. Journal of Materials Chemistry B, 3(44), 8729-8737.

Tsukada, M., Fukai, M., Miki, K., Shiraishi, T., Suzuki, T., Nishio, K., Sugita, T., Ishino, M., Kinoshita, K., Takahashi, K., Shiro, M., \& Koyama, K. (2011). Chemical Constituents of a Marine Fungus,Arthrinium sacchari. Journal of Natural Products, 74(7), 1645-1649.

Yeh, M. K., Cheng, K. M., Hu, C. S., Huang, Y. C., \& Young, J. J. (2011). Novel protein-loaded chondroitin sulfate-chitosan nanoparticles:Preparation and characterization. Acta Biomaterialia, 
7(10), 3804-3812.

Zhang, C., Ping, Q., Zhang, H., \& Shen, J. (2003). Preparation of N-alkyl-O-sulfate chitosan derivatives and micellar solubilization of taxol. Carbohydrate Polymers, 54(2), 137-141.

Zhang, X., Geng, X., Jiang, H., Li, J., \& Huang, J. (2012). Synthesis and characteristics of chitin and chitosan with the (2-hydroxy-3-trimethylammonium) propyl functionality, and evaluation of their antioxidant activity in vitro. Carbohydrate Polymers, 89(2), 486-491.

Zhong, Z., Li, P., Xing, R., \& Liu, S. (2009). Antimicrobial activity of hydroxylbenzenesulfonailides derivatives of chitosan, chitosan sulfates and carboxymethyl chitosan. International Journal of Biological Macromolecules, 45(2), 163-168. 